\title{
Dendrochilum hampelii (Coelogyninae, Epidendroideae, Orchidaceae) traded as 'Big Pink' is a new species, not a hybrid: evidence from nrITS, matK and ycfl sequence data
}

\author{
Bobby P. Sulistyo ${ }^{1,2}$, Ronny Boos ${ }^{3}$, James E. Cootes ${ }^{3}$, Barbara Gravendeel ${ }^{1,4,5}$ \\ I Naturalis Biodiversity Center, Leiden University, 2300 RA Leiden, The Netherlands 2 University of Applied \\ Sciences Arnhem and Nijmegen, 6525 EN Nijmegen, The Netherlands 3 Centre for Australian National \\ Biodiversity Research, Canberra, Australia 4 University of Applied Sciences Leiden, 2333 CK Leiden, The \\ Netherlands 5 Institute Biology Leiden, Leiden University, 2300 RA Leiden, The Netherlands
}

Corresponding author: Barbara Gravendeel (barbara.gravendeel@naturalis.nl)

Academic editor: L. Peruzzi | Received 31 July 2015 | Accepted 27 August 2015 | Published 1 October 2015

Citation: Sulistyo BP, Boos R, Cootes JE, Barbara Gravendeel B (2015) Dendrochilum hampelii (Coelogyninae, Epidendroideae, Orchidaceae) traded as 'Big Pink' is a new species, not a hybrid: evidence from nrITS, matK and $y c f$ sequence data. PhytoKeys 56: 83-97. doi: 10.3897/phytokeys.56.5432

\begin{abstract}
In 2013, an unidentified species of Dendrochilum appeared in cultivation under the commercial trade name 'Big Pink'. Using sequences of the nuclear ribosomal ITS1-5.8S-ITS2 region and of the plastid $m a t \mathrm{~K}$ and $y c f 1$ genes, we examined the phylogenetic relationships between 'Big Pink' and six other species of the phenetically defined Dendrochilum subgen. Platyclinis sect. Eurybrachium. Separate and combined analyses (using Bayesian, Maximum Likelihood and Parsimony inference) showed consistent placement of the unidentified species within a statistically well supported clade. Furthermore, the multi-copy nrITS marker showed clear distinct peaks. Thus, we found no evidence that 'Big Pink' could be a hybrid. Against this background, and further supported by species-specific mutations in (at least) nrITS and $y c f$, we formally describe 'Big Pink' as a new species under the name Dendrochilum hampelii. Morphologically, it is most similar to D. propinquum, but it differs in a number of characters. Of the two cultivated individuals available for our study, one was of unrecorded provenance. The other allegedly originated from the Philippines. Observations of the species occurring in the wild in the Philippines in the northern provinces of Bukidnon and Misamis Oriental on the island of Mindanao confirmed this.
\end{abstract}

\section{Keywords}

Dendrochilum hampelii, Molecular Phylogenetics, Orchids, the Philippines

Copyright Bobby P. Sulistyo et al. This is an open access article distributed under the terms of the Creative Commons Attribution License (CC BY 4.0), which permits unrestricted use, distribution, and reproduction in any medium, provided the original author and source are credited. 


\section{Introduction}

The largely Malesian genus Dendrochilum Blume (Blume 1825-1827: 398-399; published 1825) accommodates ca. 275 species - most of them described based on field inventories in the periods ca. 1900-1940 and ca. 1985-2000 (Pedersen 2007a). However, since the turn of the millenium, most new species of Dendrochilum have been described based on cultivated material of unrecorded provenance (cf. Pedersen 2011). Following the publication of protologues based on cultivated material, four of these species have been located in the wild (Cootes 2011). This is the case for D. coccineum H.A.Pedersen \& Gravend. (Pedersen et al. 2004), D. croceum H.A.Pedersen (Pedersen 2005), D. quinquecallosum H.A.Pedersen (Pedersen 2007b) and D. undulatum H.A.Pedersen (Pedersen 2007b). The discovery of $D$. coccineum in the Philippines confirmed a phylogeny-based hypothesis put forward by Pedersen et al. (2004). Similar phylogenetic inference of $D$. warrenii H.A.Pedersen \& Gravend. (Pedersen et al. 2004) as probably originating from the Philippines and/or Sulawesi (Pedersen et al. 2004) is still awaiting confirmation.

The formal description of new species of unknown natural distribution has undoubtedly served to stimulate the (partly successful) search for these species in the wild, thus demonstrating the relevancy of this practice-not least in a conservation context. Nevertheless, describing new species based on material in commercial trade also involves a few problems. Thus, Vermeulen et al. (2014) raised some issues of moral concern, and Pedersen (2011) emphasized that care should be taken not to accidentally describe artificial hybrids as new species. Until recently, this risk was negligible in connection with Dendrochilum since not a single Dendrochilum hybrid had been registered (cf. Pedersen 2011). However, now there are two Dendrochilum artificial hybrids registered in The International Orchid Register (http://www.apps.rhs.org.uk/horticulturaldatabase/ orchidregister/orchidregister.asp; accessed on 13 February 2015), originating from Bornean parental species. This demonstrates that human assisted hybridization between different species of Dendrochilum is indeed possible-for which reason we must consider the possibility that seemingly undescribed species suddenly appearing in cultivation could in reality be artificial hybrids.

Since chloroplast DNA is usually maternally inherited and nuclear DNA is biparentally inherited in orchids, incongruences between nuclear and plastid gene trees might indicate past events of hybridization. For example, by comparing phylogenies based on cpDNA and nrDNA, Barkman and Simpson (2002) inferred a natural hybrid origin of D. acuiferum Carr whereas Gravendeel et al. (2004) detected natural hybrids within the more distantly related genus Pleione D.Don. Further evidence for hybridization could potentially come from significant within-individual variation in multi-copy DNA markers.

This paper reports our study of an unidentified Dendrochilum (trade name: 'Big Pink') that appeared in cultivation in 2013. A live plant presented to the Hortus botanicus in Leiden carried a tag indicating a Philippinese provenance. However, as the plant came from a commercial nursery that trades much material of unknown geographic origin, we felt this provenance was in need of verification. 
Synanthous inflorescences in combination with an entire rostellum, presence of stelidia and an apical wing on the column place the study plant in the phenetically defined subgenus Platyclinis Engl. as circumscribed by Pedersen et al. (1997). Within this subgenus, a firmly attached entire labellum and a stout and straight column with stelidia but without a foot place the plant in the phenetically defined section Eurybrachium Carr ex J.J.Wood, H.A.Pedersen \& J.B.Comber (Pedersen et al. 1997). However, the morphology of 'Big Pink' does not match any previously described species in section Eurybrachium - implying that it should be formally described as a new species, provided it is not either an artificial or natural hybrid.

Altogether, we decided to examine 'Big Pink' in a molecular phylogenetic framework - and to describe it as a new species, if the results of the phylogenetic study could reject the possibility of 'Big Pink' being a hybrid.

\section{Methods}

\section{Plant sampling and DNA extraction}

The possible hybrid status of 'Big Pink' was tested using a molecular phylogenetic approach based on three markers, namely the biparentally inherited multi-copy nuclear ribosomal internal transcribed spacer (nrITS), and the maternally inherited plastid matK and ycfl genes. The ingroup consisted of 'Big Pink' and six other species belonging to Dendrochilum subgen. Platyclinis sect. Eurybrachium (cf. Pedersen et al. 1997), see Table 1.

A live plant of 'Big Pink' was available from the Hortus botanicus in Leiden, whereas the remaining Dendrochilum plants sampled for this study were reared in the Botanical Garden, Natural History Museum of Denmark. For information on vouchers, see Table 1. Thunia alba Rchb.f. was chosen as outgroup, based on the placement of the genus Thunia as sister to Dendrochilum using sequences from $\operatorname{nrTS}$, matK, $t r n \mathrm{~L}-\mathrm{F}$, and $r b c \mathrm{~L}$ (Goldman et al. 2001, Gravendeel et al. 2001, van den Berg et al. 2005).

Total genomic DNA was obtained from $50 \mathrm{mg}$ of silica dried or fresh leaf tissue. In the case of 'Big Pink', the tissue was mechanically reduced to dry powder using liquid nitrogen; for all other taxa, it was ground in Lysing Matrix A tubes (MP Biomedicals) and extracted using the DNeasy Plant Mini Kit (Qiagen) following the manufacturer's protocol.

\section{Amplification and Sanger sequencing}

The ITS1-5.8S-ITS2 region of the nuclear ribosomal internal transcribed spacer (nrITS) was amplified using primers 17SE (ACGAATTCATGGTCCGGTGAAGTGTTC) and 26SE (TAGAATTCCCCGGTTCGCTCGCCGTTAC), as described by Sun et al. (1994). Subsequently, a M13 universal sequencing primer was added to the 
Table I. List of species sampled for our DNA-based phylogenetic analysis with voucher data. All species in the table belong to the phenetically defined Dendrochilum subgen. Platyclinis sect. Eurybrachium. Abbrevations of herbaria: C=University of Copenhagen, Copenhagen, Denmark; K=Royal Botanic Gardens Kew, United Kingdom; L=Naturalis Biodiversity Center, Leiden, The Netherlands; N=Nanjing University, Nanjing, China.

\begin{tabular}{l|c|c|c}
\hline \multirow{2}{*}{$\begin{array}{l}\text { Species } \\
\text { Voucher }\end{array}$} & \multicolumn{2}{|c}{ NCBI GenBank accession numbers } \\
\cline { 2 - 4 } $\begin{array}{l}\text { Dendrochilum apoense T.Hashim. } \\
\text { cult. Hort. Bot. Hafn. s.n. (C!) }\end{array}$ & KT334200 & KT334206 & KT334213 \\
\hline $\begin{array}{l}\text { Dendrochilum auriculare Ames } \\
\text { cult. Hort. Bot. Hafn. P2012.5172 (C!) }\end{array}$ & KT334201 & KT334207 & KT334214 \\
\hline $\begin{array}{l}\text { Dendrochilum coccineum H.A.Pedersen \& Gravend. } \\
\text { cult. Richard C. Warren, Warren EQ 3060 (C!) }\end{array}$ & AY534923 & KT334208 & KT334215 \\
\hline $\begin{array}{l}\text { Dendrochilum convallariiforme Schauer } \\
\text { cult. Hort. Bot. Hafn. P2012.5177 (C!) }\end{array}$ & KT334202 & KT334209 & KT334216 \\
\hline $\begin{array}{l}\text { * Dendrochilum hampelii Sulistyo et al. } \\
\text { cult. Hort. bot. Leiden 20130654 (L! [WAG0116920]]) }\end{array}$ & KT334203 & KT334210 & KT334217 \\
\hline $\begin{array}{l}\text { Dendrochilum septemnervium H.A.Pedersen } \\
\text { cult. Hort. Bot. Hafn. P2012.5195 (C!) }\end{array}$ & KT334204 & KT334211 & KT334218 \\
\hline $\begin{array}{l}\text { Dendrochilum tortile H.A.Pedersen } \\
\text { cult. Hort. Bot. Hafn. P2012.5200 (C!) }\end{array}$ & KT334205 & KT334212 & KT334219 \\
\hline $\begin{array}{l}\text { Thunia alba } \text { (Lindl.) Rchb.f. } \\
\text { Nepal, Chase } 589 \text { (K!) }\end{array}$ & AY008466 & AY121731 & - \\
\hline $\begin{array}{l}\text { Thunia alba } \text { (Lindl.) Rchb.f. } \\
\text { China, B. Hou EThuA (N!) }\end{array}$ & - & - & KF361675 \\
\hline
\end{tabular}

*Trade name: 'Big Pink'

5' end of the forward (TGTAAAACGACGGCCAGT) and reverse (CAGGAAACAGCTATGAC) primers to improve Sanger sequencing efficiency. Each PCR reaction consisted of $25 \mu \mathrm{l}$, containing the template DNA, CoralLoad PCR buffer (Qiagen), dNTPs, Taq DNA Polymerase (Qiagen), and both primers. The PCR reactions were carried out using a MyCycler Thermal Cycler (Bio-Rad) or a C1000 Touch Thermal Cycler (Bio-Rad). The thermal cycling protocol began with $5 \mathrm{~min}$ initial denaturation at $95^{\circ} \mathrm{C}$ followed by 35 amplification cycles, each with $30 \mathrm{sec}$ denaturation at $95^{\circ} \mathrm{C}$, $30 \mathrm{sec}$ annealing at $50^{\circ} \mathrm{C}$, and $1 \mathrm{~min}$ extension at $72^{\circ} \mathrm{C}$, which were concluded by a 7 min final extension at $72^{\circ} \mathrm{C}$.

The primers for the amplification of the chloroplast mat $\mathrm{K}$ region were also used by Gravendeel et al. (2001), and specified as follows: 19F (CGTTCTGACCATATTGCACTATG) and 881R (TMTTCATCAGAATAAGAGT), 731F (TCTGGAGTCTTTCTTGAGCGA) and 2R (AACTAGTCGGATGGAGTAG). The PCR mix for the amplification of matK followed that of nrITS, but with additional BSA. The thermal cycling protocol for matK PCR began with 5 min initial denaturation at $94^{\circ} \mathrm{C}$ followed by 28 amplification cycles, each with $30 \mathrm{sec}$ denaturation at $94^{\circ} \mathrm{C}, 30 \mathrm{sec}$ annealing at $49^{\circ} \mathrm{C}, 1 \mathrm{~min}$ extension at $72{ }^{\circ} \mathrm{C}$, and ended with a $7 \mathrm{~min}$ final extension at $72^{\circ} \mathrm{C}$. 
The 3' end portion of the chloroplast $y c f$ region was amplified using primers newly designed in this study. The design was based on the $y c f 1$ sequences data set of Neubig et al. (2008) available on NCBI GenBank (http://www.ncbi.nlm.nih.gov/ GenBank), specifically from the species of subfamily Epidendroideae. The sequences were aligned using Geneious 5.6.7 (Kearse et al. 2012), and conserved regions were identified to be used as annealing sites. The $y c f 1$ sequences produced in this study had a complete marker size of approximately $1.5 \mathrm{~kb}$. The $y c f 1$ region was amplified using a Hot start PCR protocol with primers d147F (TGCAGCRAATTYATTTATGAGTC) and intR2 (GATTTGATTGGGATGATCCAAGG), d557F (TCAAGAGATCAAACCATKCAATCA) and 1560R (CTCTACGACGTCTGGGAGATAG). Each PCR reaction consisted of $25 \mu$, containing DNA template, Phire Hot Start II DNA Polymerase and Phire buffer (ThermoScientific), dNTPs, BSA, and both primers. The cycling condition started with $30 \mathrm{sec}$ initial denaturation at $98{ }^{\circ} \mathrm{C}$ and followed by 30 cycles of $5 \mathrm{sec}$ denaturation at $98^{\circ} \mathrm{C}, 5 \mathrm{sec}$ annealing at $65.5^{\circ} \mathrm{C}$, and 10 sec extension at $72{ }^{\circ} \mathrm{C}$, concluded with a 1 min final extension at $72^{\circ} \mathrm{C}$.

Sanger sequencing of the amplification products was performed at Baseclear (http://www.baseclear.com/), using an ABI 3730xl sequencer (Applied Biosystems). All new sequences are deposited in NCBI GenBank (Table 1). All sequences of T. alba were obtained from NCBI GenBank.

\section{Phylogenetic analyses}

Raw Sanger sequencing results in the form of $A B 1$ files were edited and contigged using Sequencher 5.3 sequence analysis software (http://www.genecodes.com). The ends of all data sets were trimmed to avoid character misinterpretation. Ambiguous bases were replaced with " $\mathrm{N}$ " in the data matrix. The sequences were aligned using Geneious multiple sequence alignment in Geneious 5.6.7 (Kearse et al. 2012) with subsequent manual adjustments. Missing data were replaced with "?".

Phylogenetic analyses were carried out by means of Maximum Parsimony and Maximum Likelihood using PAUP* and Bayesian methods using the software Bayesian Evolutionary Analysis and Sampling of Trees (BEAST ver. 1.8.0; Drummond et al. 2012). Both PAUP* and the BEAST program were used to analyze the nrITS, the combined plastid matK $+y c f$, and the combined nrITS + matK + ycfl data matrices. For the Bayesian analysis, the substitution and clock model was set as unlinked, and the chosen nucleotide substitution model was General Time Reversible (GTR), plus Gamma with 10 categories. The best fit substitution model for each partition was determined using the Find Model web tool (http://www.hiv.lanl.gov/content/sequence/findmodel/findmodel.html). A lognormal relaxed clock model was used for each partition, and the chosen tree prior was the Yule speciation process (Gernhard 2008). Tree samplings were generated through Markov Chain Monte Carlo (MCMC), with the number of generations set to 20,000,000 and a tree sampling for every 1000 generations. Three consecutive replicates were done to assess the consistency of the method. The three consistent replicates 
were then combined into a single matrix using LogCombiner c1.8.0 (http://beast.bio. ed.ac.uk/logcombiner) and used to search for the best probable tree using the program TreeAnnotator ver. 1.8 .0 (http://beast.bio.ed.ac.uk/TreeAnnotator) with a 20\% burnin value to avoid the reduction of posterior probability (PP) values, and visualized using FigTree ver. 1.4.2 (http://tree.bio.ed.ac.uk/software/figtree/).

\section{Results}

The nrITS sequence alignment contained 854 positions with a mean ungapped length of $834 \mathrm{bp}$. Included in the alignment were the nrITS1 (236 positions), 5.8S RNA (166 positions), and mrITS2 (253 positions) regions. In total, the number of variable sites for the included positions was 164 (19.2\%), of which 39 were potentially phylogenetically informative. Mean pairwise distances within the ingroup varied from $0.2-6.9 \%$. There were six synapomorphic indels, with a size ranging from $1-5 \mathrm{bp}$. All the sequences included in the nrITS matrix were complete except for T. alba, which lacked 110 characters.

The matK matrix was characterized by a fairly high number of missing data, mostly due to amplification failures. Samples lacking approximately half (800 bp) of the entire matK sequence included $D$. apoense T.Hashim., D. septemnervium H.A.Pedersen and $D$. tortile $\mathrm{H}$.A.Pedersen. The mat $\mathrm{K}$ alignment consisted of 1,783 positions, with a mean ungapped length of approximately $1,769 \mathrm{bp}$. The alignment contained the least variable sites out of the three alignments, 91 sites $(5.1 \%)$ with 5 potentially phylogenetically informative sites. Mean pairwise distances within the ingroup ranged from $0.8-71.1 \%$. There was a synapomorphic indel of $1 \mathrm{bp}$.

The $y c f$ alignment consisted of 1,297 positions with mean ungapped length of $1065 \mathrm{bp}$. The number of variable sites was similar to that of nrITS, 244 (18.8\%). Out of these positions, 30 were phylogenetically informative. There was one synapomorphic indel with a size of $9 \mathrm{bp}$. Mean pairwise distances within the ingroup varied from 10-33.5\%. Amplification failures for D. septemnervium resulted in almost half of the desired $y c f 1$ marker missing for this species.

The phylogenetic trees based on the combined mat $K+y c f 1$ matrix obtained by all three methods yielded overall stronger branch support relative to that of nrITS (Fig. $1 \mathrm{~A})$. The internal nodes gained high $(\mathrm{PP}=0.97-1.0)$ support values, and the preceding replicates showed consistent topologies as well as well-supported clades. Clades presented by the mat $\mathrm{K}+y c f 1$ tree were highly congruent with the nrITS tree, and with higher support value.

No hard incongruence was present between the nrITS and the plastid phylogenetic trees obtained by all three methods. The combined nrITS + matK $+y c f 1$ matrix yielded a single tree with highly consistent topology and strong support values; the same clades as those in the separate nrITS and matK $+y c f 1$ analyses were identified (Fig. 1B). In all our trees, 'Big Pink' was positioned as a sister to D. tortile and D. septemnervium, which were very closely related, in terms of relative branch length (Fig. 1). 

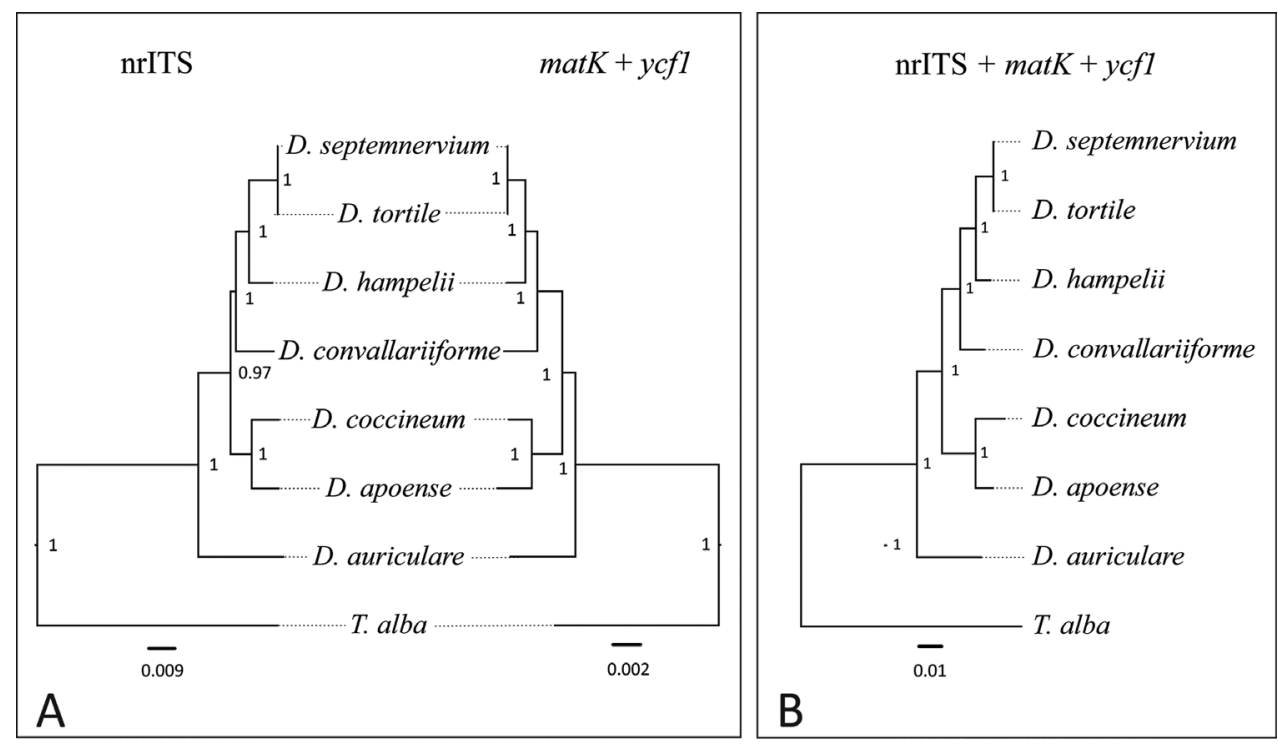

Figure I. Phylogenetic relationships amongst the sampled species of Dendrochilum, created using BEAST and PAUP*. The values on the nodes represent posterior probabilities, whereas branch lengths indicate to the relative number of changes: $\mathbf{A}$ comparison between topologies based on $\operatorname{nrITS}$ and mat $\mathrm{K}+y c f 1$ matrices B topology resulting from the combined nrITS + mat $\mathrm{K}+y c f$ data matrices.

\begin{tabular}{|c|c|c|}
\hline $\begin{array}{l}\text { Consensus } \\
\text { Identity }\end{array}$ & 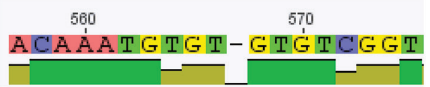 & 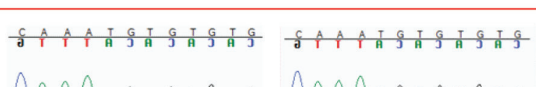 \\
\hline $\begin{array}{l}\text { 1. D. apoense } \\
\text { 2. D. auriculare } \\
\text { 3. D. coccineum } \\
\text { 4. D. convallariiforme } \\
\text { 5. D. hampelii } \\
\text { 6. D. septemnervium } \\
\text { 7.D. tortile } \\
\text { 8. T. alba }\end{array}$ & 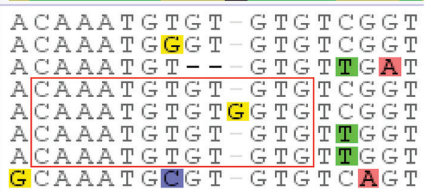 & $\frac{\text { D. convallariforme }}{\text { D. hampelii } \uparrow}$ \\
\hline
\end{tabular}

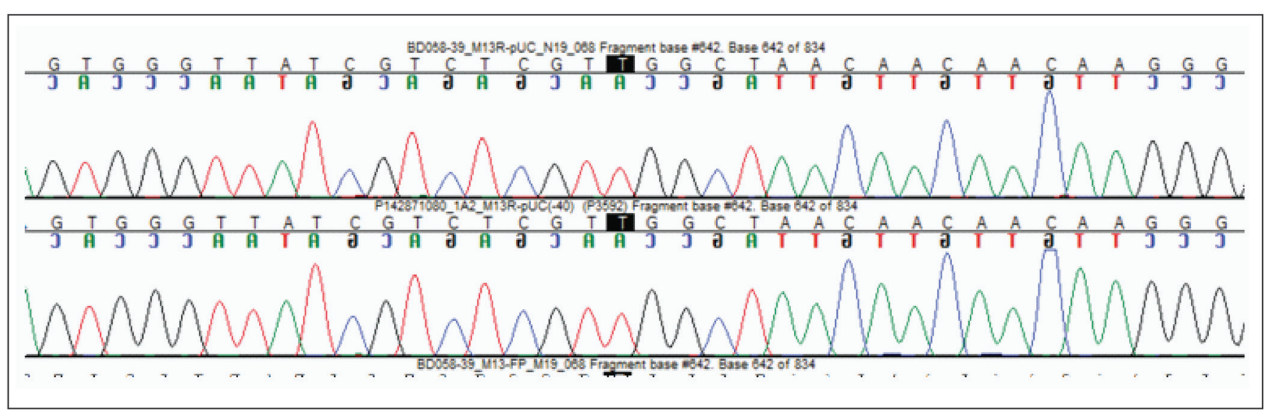

Figure 2. Above: alignment of nrITS sequences of the ingroup species from our phylogenetic analyses. For 'Big Pink' (Dendrochilum hampelii) and its apparently closest relatives among our study species, electropherograms are shown in red boxes. The electropherograms show clear distinct peaks; the speciesspecific mutation of 'Big Pink' is indicated by a red arrow. Below: electropherogram of 'Big Pink' (Dendrochilum hampelii) covering a larger region of nrITS; the distinct single peaks in both the forward and reverse sequences suggest this is a wild species rather than an artificial hybrid (see text for details). 
Analysis of the nrITS sequence alignment revealed a species-specific mutation of 'Big Pink' at position 567 (Fig. 2). Electropherograms of the ITS sequences of 'Big Pink' and its three closest relatives among the study species showed clear, distinct signals for positions included in the alignment, including the 'Big Pink' species-specific mutation site (Fig. 2).

\section{Discussion}

Nuclear sequence variation found was largely in agreement with previous studies in Coelogyninae (Gravendeel et al. 2001). In general, sequence divergence of nrITS between different species of Dendrochilum is known to vary between 0.2-20\% (Pedersen et al. 2004; Sulistyo, unpublished data). Additionally, the nrITS sequence obtained from $D$. hampelii is not likely to be a paralogous locus since branch lengths are similar to the sequences of other bona fide species analyzed. The position of 'Big Pink' was in agreement with preliminary analyses based on larger sampling (Sulistyo, unpublished data), as well as with the analyses based on different sequences. We found no evidence suggesting that the morphologically distinctive 'Big Pink' could be an artificial hybrid rather than a new wild species. Firstly, the separate phylogenetic analyses of nuclear and plastid markers proved highly congruent. Secondly, the electropherogram of the nrITS sequence of 'Big Pink' showed distinct single peaks with no indication of any heterozygosity of the specimen examined.

Genetically, 'Big Pink' possessed a number of automorphic mutations compared to the other species included in our small phylogenetic study. These unique mutations were found in nrITS and $y c f l$, whereas one in matK is in need of verification due to the alignment being characterized by a fairly high number of missing data. Morphologically, 'Big Pink' is most similar to D. propinquum Ames. Unfortunately, no material was available of $D$. propinquum for DNA sequencing for this study. However, 'Big Pink' has much larger flowers (approximately twice the size of those of $D$. propinquum), its flowers have petals that are 1.4-1.5 times as broad as the sepals $(0.8-1.1$ times in $D$. propinquum), its labellum is broadly cordate (broadly elliptic to ovate in D. propinquum), and the stelidia of its column are acute (obtuse in D. propinquum). Against this background, we describe 'Big Pink' as a new species below.

Contrary to the studies of Wanntorp et al. (2002) and Pedersen et al. (2004), the taxon sampling underlying the phylogenetic analyses in this study was too small to allow for any inference of a probable geographic origin of 'Big Pink'. However, in a preliminary study based on systematically and geographically much broader sampling of Dendrochilum s.l., individual phylogenetic analyses using sequences from nrITS, plastid $m a t \mathrm{~K}$, and $y c f 1$ have all indicated 'Big Pink' to be nested within a clade nearly exclusively consisting of Philippine endemics (Sulistyo, unpublished data). In subsequent field surveys by the second author, a total of 12 plants were observed in February 2014 in the northern provinces of Bukidnon and Misamis Oriental on the island of Mindanao at high elevation, a few of which were flowering. In March 2015, only 4 
individuals were observed in situ in the wild by the second and third author, and none were flowering. These observations confirm that $D$. hampelii is indeed a Philippinese, almost certainly endemic species. It is conjectured to have entered cultivation in Europe through the many domestic markets in Southeast Asia that sell orchid species. For these markets, wild plants are harvested but traded as 'cultivated' to circumvent CITES legislation. All Coelogyninae are listed on Appendix 2. Despite this legal protection, illegal trade is continuing at international orchid shows and by web based orders from buyers of specific species or nursery owners hoping to incorporate desirable wild traits into new hybrids (Hinsley et al. 2015).

\section{Taxonomic treatment}

Based on these results, it is determined that "Big Pink" is a new species in need of recognition. Formally naming the species is relevant for horticulture and ex situ conservation, because the name provides an unambiguous way to refer to the species. The morphology of 'Big Pink' was described using terminology of the vocabulary and list of individual absolute terms in Stearn (1983), if relevant standardized according to the Orchidaceae glossary in Pedersen et al. (2011).

\section{Dendrochilum hampelii Sulistyo, Gravend., R.Boos \& Cootes, sp. nov.} urn:Isid:ipni.org:names:77150223-1

Figs 3, 4

Type. Sine loco et anno, Perry 490 (holotype L!).

Diagnosis. This new species is similar to $D$. propinquum Ames, but is distinguished by its larger flowers with petals proportionally broader $(1.4-1.5 \times)$ than the sepals, a broadly cordate labellum $(6.8-8.0 \times 7.2-7.6 \mathrm{~m})$ and acute stelidia.

Medium-sized, tufted epiphytic herb. Roots appearing from the rhizome, ca. 2.7 $\mathrm{mm}$ in diameter. Pseudobulbs tightly clustered on a short rhizome, fusiform, 3.5-5.0 $\mathrm{cm}$ long, $0.5-1.4 \mathrm{~cm}$ in diameter, longitudinally striated when dry, 1-leaved, initially covered by ca. 3 imperfectly to nearly perfectly tubular, rounded to acute cataphylls that soon disintegrate into persistent fibers. Leaves convolute, dorsiventrally flattened, petiolate; petiole channeled, $3.0-4.5 \mathrm{~cm}$ long; lamina (ob)lanceolate, obtuse, 13.0 $20.0 \times 3.7-5 \mathrm{~cm}$, subcoriaceous, with 7-8 distinct (and many indistinct) nerves. Inflorescence synanthous, racemose; peduncle suberect, arched, slender, somewhat flattened, $18.0-21.2 \mathrm{~cm}$ long, sparsely and finely setose; rachis pendent with distichously alternating flowers (but the rachis axis twisted so as to produce a cylindrical inflorescence), many-flowered with internodes of 3-7 mm, somewhat furrowed, $20.0-27.5 \mathrm{~cm}$ long, sparsely and finely setose, basally with 1 appressed non-floriferous bract; flowering starting from the proximal part of the rachis. Floral bracts glumaceous, broadly lanceolate to (ovate-)oblong when flattened, obtuse to acute, $4.0-9.5 \times 2.2-4.3 \mathrm{~mm}$, entire, 


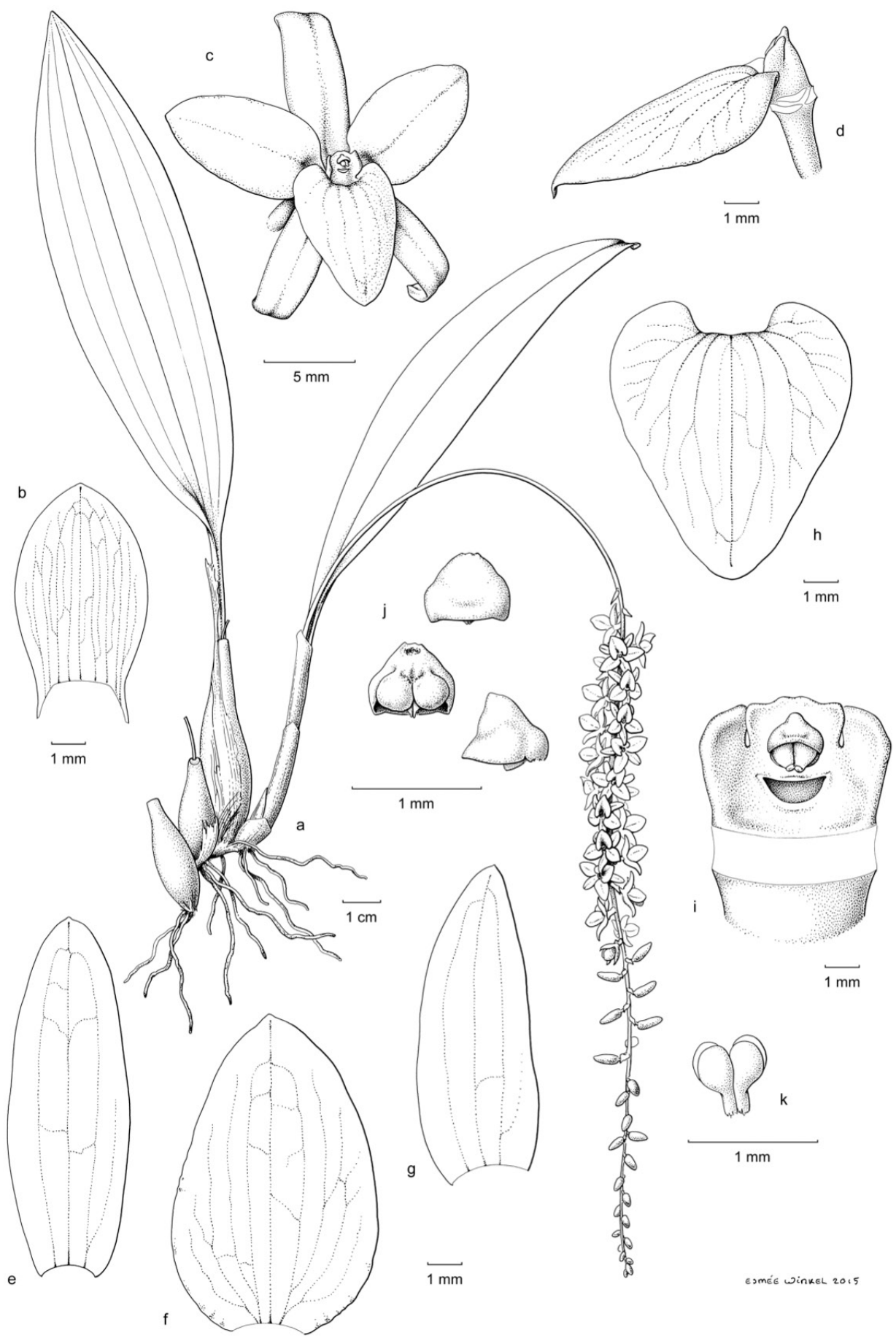

Figure 3. Dendrochilum hampelii: a habit $\mathbf{b}$ floral bract $\mathbf{c}$ flower $\mathbf{d}$ flower (sepals and petals removed) e dorsal sepal $\mathbf{f}$ petal $\mathbf{g}$ lateral sepal $\mathbf{h}$ labellum $\mathbf{i}$ column, front view $\mathbf{j}$ anther $\mathbf{k}$ pollinia. Drawing by Esmee Winkel based on Hort. bot. Leiden 20130654 (L! [spirit no. WAG0116920]). 


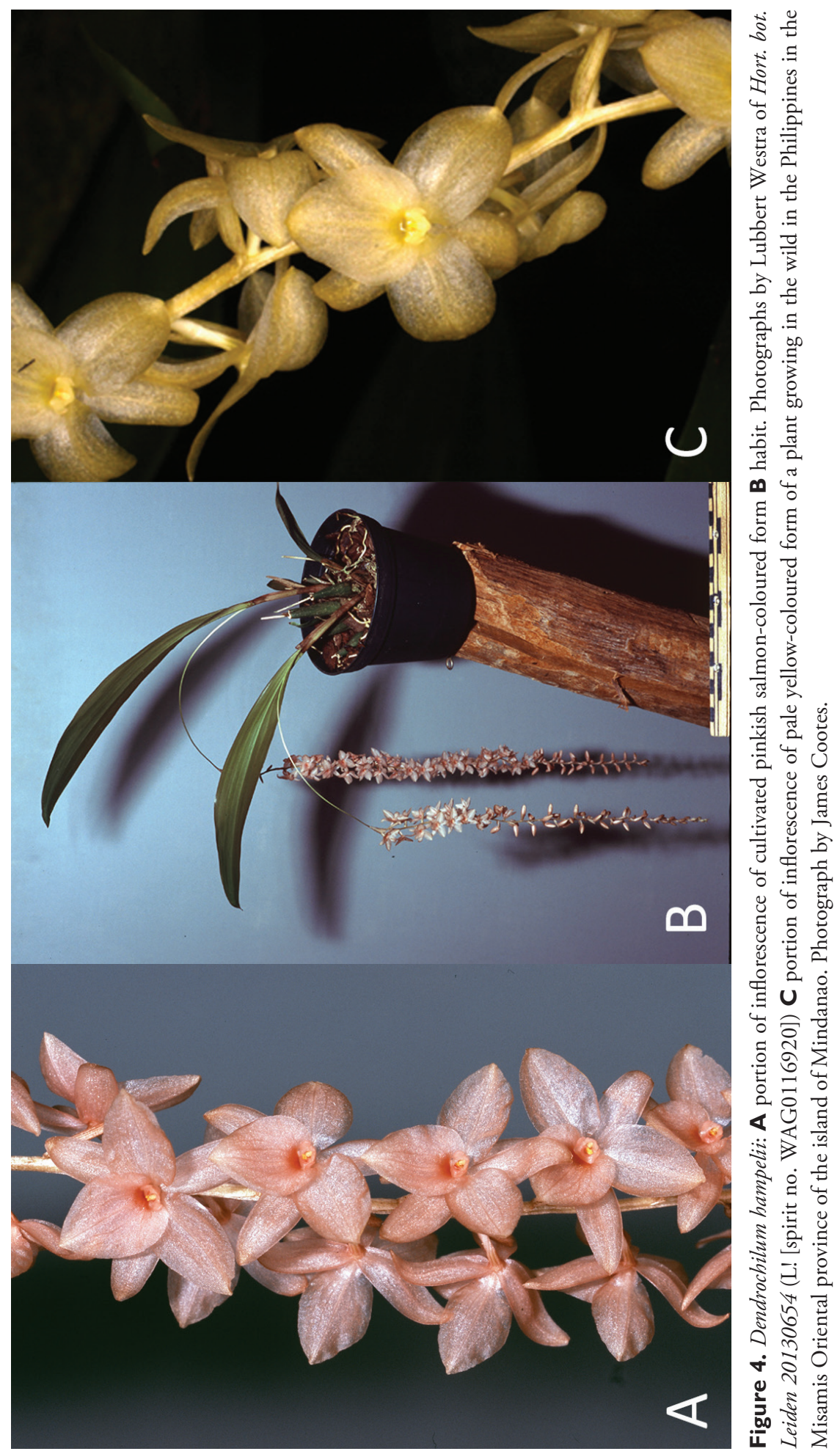


9- to 19-nerved from the base, finely setose on the dorsal side. Flowers non-resupinate, pinkish salmon-coloured (Fig. 4A-B) or pale yellow (Fig. 4C) with yellow anther. Sepals recurved with revolute margins, entire, obtuse to rounded, minutely mucronate, glabrous, 3- to 5-veined from the base; dorsal sepal lanceolate-oblong, 8.7-11.0 $\times$ $3.5-3.7 \mathrm{~mm}$ when flattened; lateral sepals ovate-oblong, slightly oblique, 8.1-10.0 $\times$ $3.7-4.0 \mathrm{~mm}$ when flattened. Petals recurved with flat margins, broadly (ovate-)elliptic, often with a subbasal fold in either side, rounded to acute, 8.4-11.1 $\times 5.4-5.7 \mathrm{~mm}$, 1.4-1.5 times as wide as the sepals, entire, glabrous, 3- to 5-veined from the base. $\mathrm{La}$ bellum firmly attached, sessile, describing a right to obtuse angle to the column, flat, broadly cordate with entire margins, acute to short-acuminate, $6.8-8.0 \times 7.2-7.6 \mathrm{~mm}$, without ornaments, glabrous and smooth, 5- to 7-veined from the base. Column suberect, straight, semiterete, $1.7-2.1 \mathrm{~mm}$ long, smooth, distally prolonged into a truncate to obscurely 3-lobed wing that distinctly exceeds the anther; stelidia appearing from the distal part of column proper, erect, falcately triangular-oblong, acute, subequal to the apical wing; anther circular to transversely elliptic in upper view, rounded in front, lobed at the back, with a small wart on top; pollinia 4, ellipsoid, devoid of caudicles; rostellum slightly protruding, flat, semicircular; fertile stigma part crescentshaped, concave. Ovary (including pedicel) subterete, slightly longitudinally furrowed, twisted through $180^{\circ}$, distally incurved, $3.8-4.5 \mathrm{~mm}$ long, glabrous. Fruit not seen.

Additional material examined. PHILIPPINES? Sine loco et anno, sine coll./cult. Hort. bot. Leiden 20130654 (L! [spirit no. WAG0116920]).

Etymology. The specific epiphet honours Georg Hampel, who was one of the first to provide us with study material of the newly described species.

Distribution and ecology. The species occurs in the wild in the Philippines in the northern provinces of Bukidnon and Misamis Oriental on the island of Mindanao (Fig. 4C). It grows as an epiphyte at elevations approximately $1,200 \mathrm{~m}$ above sea level among mosses on the trunks and branches of trees. Fresh flowers of plants observed in the wild were pale yellow whereas fresh flowers of the cultivated plants studied were pinkish salmon-coloured. We do not consider this reason to describe them as a different variety or forma as color dimorphism is known to occur in other Coelogyninae as well (Gravendeel 2000).

Reproductive biology. The live plant in Leiden flowered in mid-December. Attempts to pollinate flowers of $D$. hampelii were made using pollinia from the same flower and pollinia from a different flower in the same inflorescence. None of these efforts led to fruit formation. This indicates that $D$. hampelii is probably self-incompatible, as previously demonstrated for D. longibracteatum Pfitzer (Pedersen 1995), although it should be noted that experimental pollination was severely challenged by the small size of the stigmatic cavity.

Conservation status. Although the species occurs in cultivation we as yet know very little about the distribution and abundance of $D$. hampelii in the wild. As such, we recommend the species to be considered for the Data Deficient category of the IUCN Red List of Threatened Species (IUCN 2012). 


\section{Acknowledgements}

We thank Henrik Æ. Pedersen for his feedback on earlier drafts of this manuscript and Esmee Winkel and Lubbert Westra for preparing the line drawing and part of the photos, respectively, and for allowing us to publish these illustrations. Additionally, we are grateful to Georg Hampel, Malcolm Perry and Koos Wubben for providing plant material and to Gitte Petersen and Charlotte Hansen for providing DNA samples from the Natural History Museum of Denmark. The first author thanks Nelleke Kreike for supervision; Marcel Eurlings, Adam Karremans, Rachel Schwallier, Rogier R. van Vugt, and Jaco Kruizinga for other help that included checking of the English; and journal subject editor Clifford Morden for comments and advices.

\section{References}

Ames O (1920) Orchidacex. Illustrations and studies of the family Orchidacex 6. The Merrymount Press, Boston, 1-335.

Barkman TJ, Simpson BB (2002) Hybrid origin and parentage of Dendrochilum acuiferum (Orchidaceae) inferred in a phylogenetic context using nuclear and plastid sequence data. Systematic Botany 27: 209-220.

Bentham G (1881) Notes on Orchideae. Journal of the Linnean Society, Botany 13: 281-360. doi: 10.1111/j.1095-8339.1881.tb01258.x

van den Berg C, Goldman DH, Freudenstein JV, Pridgeon AM, Cameron KM, Chase MW (2005) An overview of the phylogenetic relationships within Epidendroideae inferred from multiple DNA regions and recircumscription of Epidendreae and Arethuseae (Orchidaceae). American Journal of Botany 92: 613-624. doi: 10.3732/ajb.92.4.613

Blume CL (1825-1827) Bijdragen tot de flora van Nederlandsch Indië. Jakarta, 1-1169.

Carr CE (1935) Two collections of orchids from British North Borneo, part I. Gardens' Bulletin Straits Settlements 8: 165-240.

Cootes J (2011) Philippine native orchid species. Katha Publishing Co., Quezon City, 1-289. Don D (1825) Prodromus floræ nepalensis, sive enumeratio vegetabilium, quæ in itinere per Nepaliam proprie dictam et regiones conterminas, ann. 1802-1803. J. Gale, London, $1-256$.

Drummond AJ, Suchard MA, Xie D, Rambaut A (2012) Bayesian phylogenetics with BEAUti and the BEAST. Molecular Biology and Evolution 29: 1969-1973. doi: 10.1093/molbev/ mss075

Engler A (1907) Das Pflanzenreich. Regni vegetabilis conspectus, IV 50 II B 7. Verlag von Wilhelm Engelmann, Leipzig, 1-169.

Gernhard T (2008) The conditioned reconstructed process. Journal of Theoretical Biology 253: 769-778. doi: 10.1016/j.jtbi.2008.04.005

Goldman DH, Freudenstein JV, Kores PJ, Molvray M, Jarrell DC, Whitten MW, Cameron KM, Jansen RK, Chase MW (2001) Phylogenetics of Arethuseae (Orchidaceae) based on plastid $m a t K$ and $r b c L$ sequences. Systematic Botany 26: 670-695. 
Gravendeel B (2000) Reorganising the orchid genus Coelogyne - a phylogenetic classification based on molecules and morphology. Grafische Vormgeving Kanters, Sliedrecht, 1-208.

Gravendeel B, Chase MW, de Vogel EF, Roos MC, Mes THM, Bachmann K (2001) Molecular phylogeny of Coelogyne (Epidendroideae; Orchidaceae) based on plastid RFLPs, matK, and nuclear ribosomal ITS sequences: evidence for polyphyly. American Journal of Botany 88: 1915-1927. doi: 10.2307/3558367

Gravendeel B, Eurlings MCM, van den Berg C, Cribb PJ (2004) Phylogeny of Pleione (Orchidaceae) and parentage of its wild hybrids based on plastid and nuclear ribosomal ITS sequences and morphological data. Systematic Botany 29: 50-63. doi: $10.1600 / 036364404772973988$

Hashimoto T (1981) A new species of the genus Dendrochilum Bl. (Orchidaceae) from the Philippines. Journal of Japanese Botany 56: 335-338.

Hinsley A, Verissimo D, Roberts DL (2015) Heterogeneity in consumer preferences for orchids in international trade and the potential for the use of market research methods to study demand for wildlife. Biological Conservation 190: 80-86. doi: 10.1016/j.biocon.2015.05.010

IUCN (2012) IUCN red list of threatened species categories and criteria, vers. 3.1. Second edition. IUCN, Gland, Switzerland and Cambridge, UK, iv + 32 pp.

Kearse M, Moir R, Wilson A, Stones-Havas S, Cheung M, Sturrock S, Buxton S, Cooper A, Pedersen HÆ (1995) Anthecological observations on Dendrochilum longibracteatum-a species pollinated by facultatively anthophilous insects. Lindleyana 10: 19-28.

Markowitz S, Duran C, Tierer T, Ashton B, Mentjies P, Drummond A (2012) Geneious Basic: an integrated and extendable desktop software platform for the organization and analysis of sequence data. Bioinformatics 28: 1647-1649. doi: 10.1093/bioinformatics/bts199

Neubig KM, Whitten MW, Carlsward BS, Blanco MA, Endara L, Williams NH, Moore M (2008) Phylogenetic utility of $y c f 1$ in orchids: a plastid gene more variable than $m a t \mathrm{~K}$. Plant Systematics and Evolution 277: 75-84. doi: 10.1007/s00606-008-0105-0

Pedersen HÆ (1995) Anthecological observations on Dendrochilum longibracteatum —a species pollinated by facultatively anthophilous insects. Lindleyana 10: 19-28.

Pedersen HÆ (2005) A new miniature Dendrochilum. Orchid Review 113: 286-287.

Pedersen HÆ (2007a) Hotspots of narrow endemism: adequate focal points for conservation in Dendrochilum (Orchidaceae). Lankesteriana 7: 83-92.

Pedersen HÆ (2007b) Changes to Dendrochilum. Orchid Review 115: 220-226.

Pedersen HÆ (2011) Three new species of Dendrochilum, with notes on the practice of formally describing cultivated species of unknown provenance. Malesian Orchid Journal 7: $117-124$.

Pedersen HÆ, Gravendeel B, Mudiana D (2004) Three new species of Dendrochilum (Orchidaceae) and their phylogenetic positions according to plastid and nuclear ribosomal ITS sequences. Blumea 49: 351-360. doi: 10.3767/000651904X484315

Pedersen HÆ, Kurzweil H, Suddee S, Cribb PJ (2011) Orchidaceae 1 (Cypripedioideae, Orchidoideae, Vanilloideae). In: Santisuk T, Larsen K (Eds) Flora of Thailand 12(2). The Forest Herbarium, Department of National Parks, Wildlife and Plant Conservation, Bangkok, 1-302, Pls. I-XXIV. 
Pedersen HÆ, Wood JJ, Comber JB (1997) A revised subdivision and bibliographical survey of Dendrochilum (Orchidaceae). Opera Botanica 130: 1-85.

Reichenbach HG (1852) Gartenorchideen. III. Botanische Zeitung 10: 761-772.

Stearn WT (1983) Botanical Latin: history, grammar, syntax, terminology and vocabulary. 3rd ed. David \& Charles, Newto Abbot, xiv, 1-566.

Sun Y, Skinner DZ, Liang GH, Hubert SH (1994) Phylogenetic analysis of Sorghum and related taxa using internal transcribed spacers of nuclear ribosomal DNA. Theoretical and Applied Genetics 89: 26-32. doi: 10.1007/BF00226978

Vermeulen JJ, Phelps J, Thavipoke P (2014) Notes on Bulbophyllum (Dendrobiinae; Epidendroideae; Orchidaceae): two new species and the dilemmas of species discovery via illegal trade. Phytotaxa 184: 12-22. doi: 10.11646/phytotaxa.184.1.2

Wallich N (1830-1831) Plantae asiaticae rariores; or, descriptions and figures of a select number of unpublished East Indian plants 2. Treuttel and Würtz, London, 1-86.

Wanntorp L, Wanntorp HE, Källersjö M (2002) The identity of Gunnera manicata Linden ex Andre - resolving a Brazilian-Colombian enigma. Taxon 51: 493-497. doi: 10.2307/1554861 